\title{
The influence of preventive multiple micronutrients supplementation on liver steatosis in high-cholesterol fed C57BL6/N mice
}

\author{
Torricelli P. ${ }^{1, *}$, Ferorelli P. ${ }^{1}$, De Martino A. ${ }^{2}$, Antonelli F. ${ }^{2}$, Beninati S. ${ }^{2}$ \\ ${ }^{1}$ Department SPES, University of Molise, Campobasso, Italy \\ ${ }^{2}$ University of Rome, Tor Vergata, Dept Biology, Roma, Italy
}

Email address:

beninati@bio.uniroma2.it(Beninati S.)

\section{To cite this article:}

Torricelli P., Ferorelli P., De Martino A., Antonelli F., Beninati S.. The Influence of Preventive Multiple Micronutrients Supplementation on Liver Steatosis in High-cholesterol Fed C57BL6/N Mice. American Journal of Life Sciences. Vol. 1, No. 2, 2013, pp. 55-60 doi: 10.11648/j.ajls.20130102.16

\begin{abstract}
Liver steatosis development was obtained after high cholesterol diet (HCD) in C57BL6/N mice. Mice were preliminarily treated for 10 days with multiple micronutrients contained in a commercial food supplement called Citozym (CIZ), and successively for 40 days with CIZ and HCD, to compare the protection effect of used compounds on liver metabolism and metabolic steatosis. At the end of the experiments, livers were dissected for histological examinations. Plasma total cholesterol (TCH), triacylglycerol (TAG) aspartate aminotransferase (AST), and alanine aminotransferase (ALT) concentrations were significantly higher in all HCD-fed mice. These data support a lipotoxic model of cholesterolmediated hepatic steatosis. We found that the administration of CIZ to HCD-mouse model of steatosis significantly decreased plasma TCH, TAG, ALT and AST levels along with a reduction of the accumulated fat and inflammation in the liver. These findings suggest that a preventive and continuative treatment of CIZ exerts a protective effect against metabolic hepatosteatosis.
\end{abstract}

Keywords: Micronutrients, Antioxidants, Cholesterol, NAFLD, Metabolic Steatosis

\section{Introduction}

The typical diet in modern industrialized societies is high-fat content. Chronic consumption of this type of diet is considered a major cause of a variety of health problems including obesity, diabetes and cardiovascular diseases [1]. It is well known that liver is the main organ for lipid metabolism and its lipid content and composition depend on the diet. In fact, feeding with high-fat diet for long periods of time can have adverse effects on liver and onset of nonalcoholic fatty liver disease (NAFLD) which is one of the leading causes of hepatic dysfunction in the modern world and includes a broad spectrum ranging from benign hepatic steatosis to cirrhosis [2]. Regardless of etiology of NAFLD, lipid accumulation and oxidative stress are two requisites for this disease progression [3]. Increased fat in the liver, as a consequence of increased synthesis and decreased oxidation of fat by hepatic parenchymal cells, perturbs the metabolism of the liver, while administration of a diet rich of antioxidants may have a positive influence upon the same process [4]. Fundamental importance for the activities of cell membranes is their fluidity, which depends upon the levels of cholesterol and the length of residue chains of unsaturated fatty acids contained in phospholipids. A diet rich in cholesterol may cause disturbance of function of cell membranes with an increased or decreased activity of liver enzymes. [5]. While simple steatosis is accepted as a benign state, steatohepatitis, because of elevated liver enzymes [alanine aminotransferase (ALT: EC 2.6.1.8) and aspartate aminotransferase (AST: EC 2.6.1.1)] and certain metabolic abnormalities such as diabetes, obesity, dyslipidemia, hypertension and insulin resistance is assessed as a serious condition [6]. Therefore, we considered as markers of steatosis these two enzymes related to hepatic metabolism, which are increased in the blood in the case of damage to hepatic cells. Recently, there has been an increased interest in the recognition of natural or alternative medications in the prevention of many diseases. Despite this growing 
popularity, there is limited evidence for the effectiveness of many of these natural treatments such as antioxidants.

Antioxidants are believed to protect body cells from damage caused by by-products of oxidation, known as oxygen free radicals, which may suppress immune function [7]. These antioxidants are abundant in CIZ and have been reported to possess various health benefits [8]. The present study was undertaken to investigate whether oral dosing with a commercial mixture of antioxidants called Citozym (CIZ) rich in vitamins B5, B9, C and D, influences enzymatic activity and the metabolism of the liver in HCDfed C57BL6/N mice

\section{Materials and Methods}

Male C57BL6/N mice were purchased from IFFA Credo (L'Abreole, France). Mice were housed throughout the experiments in air-conditioned animal room and fed with a AIN-76 purified rodent diet composition (Dyets, Inc. Bethlehem, PA USA). Ten mice were used for each experimental group. Treated mice were fed ad libitum with food containing cholesterol (2 mg/g b.w./24 h ). The commercial mixture of antioxidants (CIZ) was a gift from Citozeatec (Citozeatec S.r.l. Peschiera Borromeo, Milano, Italy). Cholesterol was purchased from Sigma-Aldrich (St. Louis, MO, USA). ALT, AST, TCH and LDH were determined by kinetic methods, using kits by AlphaDiagnostic (Germany).

\subsection{Experimental Protocol}

Male C57BL6/N mice $(n=40)$ of 13 weeks of age and a body weight of about 25/35 g were divided in four groups of 10 animals each. Group A: animals on regular, standard diet as control. Group B: animals staying on a HCD for 40 days $(50 / 70 \mathrm{mg} / 24 \mathrm{~h})$. Group $\mathrm{C}$ : animals were treated by oral administration of $15 \mathrm{~mL} /$ day of CIZ, diluted $1: 2 \mathrm{v} / \mathrm{v}$ with physiological solution (PS) for 60 days and subjected to HCD from day 11 to day 40. Group D: animals were treated by oral administration of $15 \mathrm{~mL} /$ day of $\mathrm{CIZ}$, diluted 1:4 v/v with PS for 60 days and subjected to HCD from day 11 to day 40. These doses were selected from previous dose-response studies [9]. Briefly, treatments with CIZ were started 10 days prior to the treatment with HCD and continued for the following 40 days. 24 hours after the last treatment with CIZ plus HCD, C57BL6/N mice were sacrificed under anesthesia, with pentobarbital $60 \mathrm{mg} / \mathrm{kg}$ and their livers were dissected for histological examination. Plasma for biochemical analysis was collected every 4 days during the treatments and stored at $-75^{\circ} \mathrm{C}$ until analyzed. To obtain the blood plasma, EDTA was used as anticoagulant.

\subsection{Liver Histology}

Liver sections were formalin fixed and paraffin embedded prior to sectioning. All sections were then stained with hematoxylin and eosin (Sigma), encoded, and assessed for steatosis and inflammation.

\subsection{Statistical Analyses}

The statistical analysis was performed using the MannWhitney U test for intergroup comparisons. Spearman's rank correlation test and the linear regression analysis were performed to analyze correlations between groups $\mathrm{B} / \mathrm{C}$ and $\mathrm{B} / \mathrm{D}$ and the duration of administration of CIZ and to draw least squares regression lines. Values were expressed as the mean \pm SD. $p<0.05$ was regarded as statistically significant.

\section{Results}

\subsection{Effects of CIZ on HCD-induced Steatosis in C57BL6/N Mice}

As shown in Figure 1, the TCH of C57BL6/ $\mathrm{N}$ mice of the four groups of treatment taken at the end of the study were statistically different. The TCH of mice of the control (group A) was found about $6.64 \pm 2.19 \mathrm{mmol} / \mathrm{L}$, during the time of experiments. TCH of mice under HCD (group B), increased during the time and reached in about 40 days of treatment a value of $40.00 \pm 6.14 \mathrm{mmol} / \mathrm{L}$ and in 60 days $68,53 \pm 7.40 \mathrm{mmol} / \mathrm{L}$. The TCH of the groups $\mathrm{C}$ and D was significantly lower than that of the group B $(p<0.05)$. Data collected for these groups at 40 and 60 days of treatments were $20.20 \pm 5.43 \mathrm{mmol} / \mathrm{L}$ and $28.62 \pm 4.32 \mathrm{mmol} / \mathrm{L}$ for group $\mathrm{C}$ and $30.23 \pm 4.32 \mathrm{mmol} / \mathrm{L}$ and $57.42 \pm 4.32$ $\mathrm{mmol} / \mathrm{L}$ for group $\mathrm{D}$, respectively.

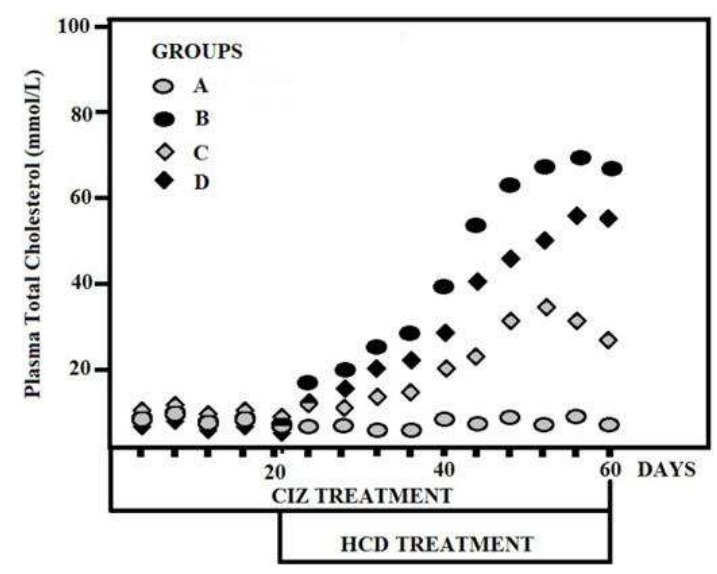

Figure 1. Plasma levels of TCH in HCD-fed and CIZ-treated C57BL6/N mice. Data are the mean of three determinations. $\pm S D$ is cited into the text.

Plasma TAG levels were significantly increased upon HCD treatment compared to the control group A $(0.62 \pm$ $0.12 \mathrm{mmol} / \mathrm{L}$ ) (Figure 2). Indeed, the amount of plasma TAG was found at 40 and 60 days of treatments of $4.10 \pm$ $1.35 \mathrm{mmol} / \mathrm{L}$ and $6.63 \pm 2.25 \mathrm{mmol} / \mathrm{L}$, respectively. TAG of mice under HCD and CIZ treatments, decreased during the time and was at 40 days of $1.70 \pm 0.54 \mathrm{mmol} / \mathrm{L}$ and at 60 days of $1.25 \pm 0.30 \mathrm{mmol} / \mathrm{L}$ for group $\mathrm{C}$. The effect of CIZ for group D still showed a significative reduction of activity $((\mathrm{p}<0.05)$. Indeed, the TAG levels were found of 
$2.80 \pm 1.32 \mathrm{mmol} / \mathrm{L}$ at 40 days and $2.63 \pm 1.84 \mathrm{mmol} / \mathrm{L}$ at 60 days of treatments.

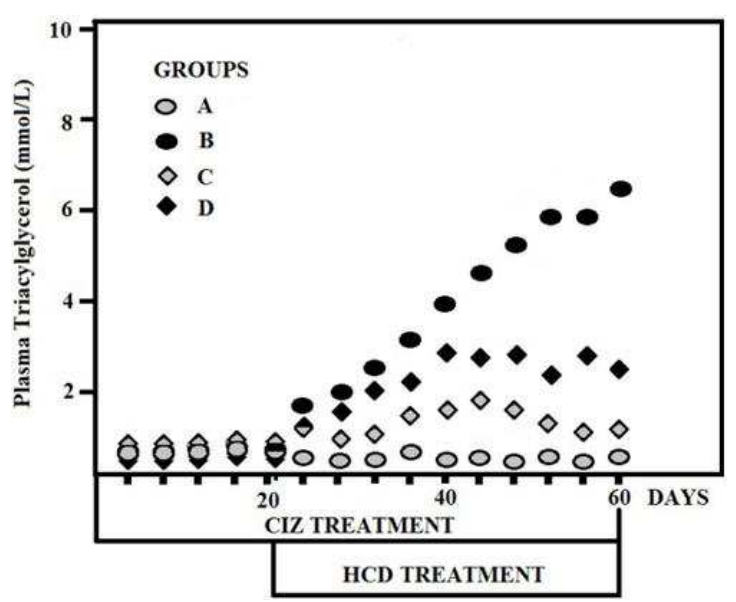

Figure 2. Plasma levels of TAG in HCD-fed and CIZ-treated C57BL6/N mice. Data are the mean of three determinations. $\pm S D$ is cited into the text

Figure 3 shows the levels of plasma ALT express as $\mathrm{IU} / \mathrm{L}$ in control cells $(4.52 \pm 2.24 \mathrm{IU} / \mathrm{L})$ and during the treatment with HCD and CIZ. HDC treatments increased the levels of ALT with a peak at 44 days of $15.00 \pm 3.75$ IU/L. The concentration suddenly decreased in 26 days to $11.53 \pm 3.32 \mathrm{IU} / \mathrm{L}$. Plasma concentration of ALT in mice under HCD and CIZ treatments, decreased during the time and was found at 40 days of $8.77 \pm 1.54 \mathrm{IU} / \mathrm{L}$ and at 60 days of $4.22 \pm 0.60 \mathrm{IU} / \mathrm{L}$ for group C. The amount of plasma ALT for group D was found at 40 and 60 days of treatments of $11.70 \pm 2.35 \mathrm{IU} / \mathrm{L}$ and $8.43 \pm 2.25 \mathrm{IU} / \mathrm{L}$, respectively.

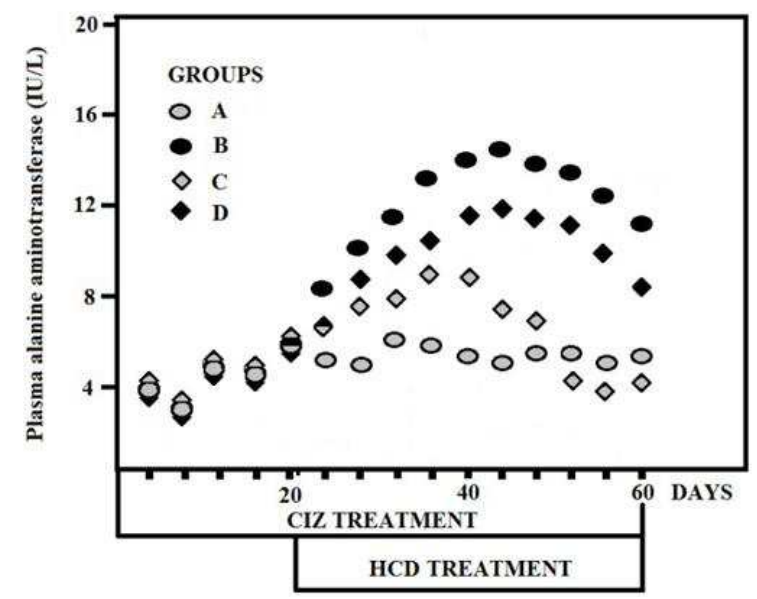

Figure 3. Plasma levels of ALT in HCD-fed and CIZ-treated C57BL6/N mice. Data are the mean of three determinations. $\pm S D$ is cited into the text

Figure 4 shows the levels of plasma AST express as IU/L in control cells $(3.22 \pm 1.44 \mathrm{IU} / \mathrm{L})$ and during the treatment with HCD and CIZ. HDC treatments increased the levels of AST with a peak at 40 days of about $12.70 \pm$ $2.65 \mathrm{IU} / \mathrm{L}$ that slightly decreased in 10 days to a value of $11.21 \pm 2.21 \mathrm{IU} / \mathrm{L}$. The mice under treatment with HCD and CIZ, showed a reduction of AST at 40 and 60 days for both groups $\mathrm{C}$ and D. The activity of CIZ for group C was more effective in AST plasma levels reduction (6.23 \pm 1.63 at 40 days; $5.12 \pm 2.12$ at 60 days) than in group D (10.50 \pm 2.31 at 40 days; $8.2 \pm 1.77$ at 60 days).

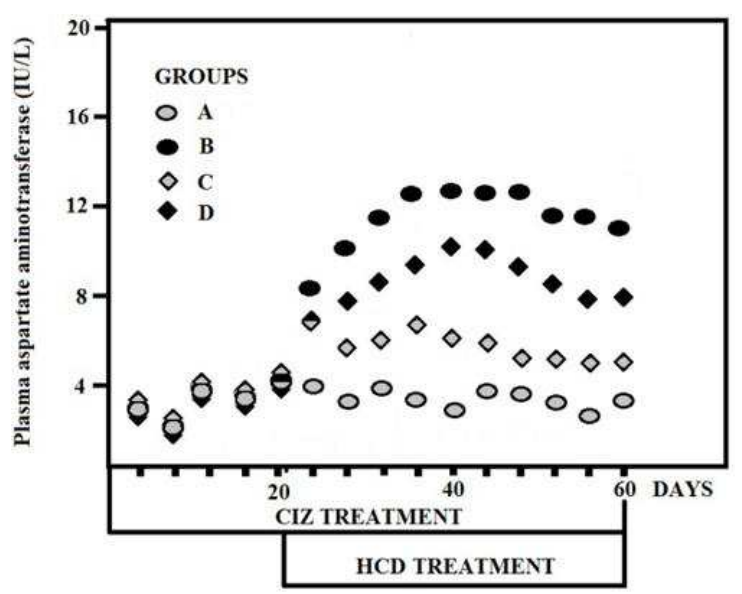

Figure 4. Plasma levels of AST in HCD-fed and CIZ-treated C57BL6/N mice. Data are the mean of three determinations. $\pm S D$ is cited into the text.

\subsection{Effects of Treatments with CIZ on Liver of HCD-fed C57BL6/N Mice}

The Histological sections of normal liver tissue are shown in Figure 5a. HCD-fed mice showed predominantly large lipid-filled vacuoles (Figure 5b). Liver sections from HCD and 1:2 CIZ-fed mice revealed a reduction of lipid accumulation in the form of lipid droplets, or even small lipid droplets (Figure 5c). Evaluation of hepatic inflammation with hematoxylin and eosin liver staining, revealed significant differences between $\mathrm{HCD}$ and $\mathrm{HCD}$ and CIZ-fed mice (data not shown). Indeed CIZ treatment led to a reduction of the accumulated fat and of inflammation in the liver. These observations indicate the preventive effects of CIZ on HCD-induced steatosis.

\section{Discussion}

Oxidative stress is considered to play a central role in the pathogenesis of NAFLD because the increased production of ROS is known to cause lipid peroxidation, followed by activation of the inflammatory response, and of stellate cells, leading to fibrogenesis [10-11]. Lipid peroxidation usually leads to the formation of peroxyl radicals, which are the central species of the peroxidation chain reaction.

In Japan, acute encephalopathy with hepatic steatosis resembling Reye's syndrome has been reported to occur after treatment with the pantothenic acid (Vitamin B5) antagonist, calcium hopantenate [12]. Pantethine is a dimeric form of pantothenic acid, composed of two molecules of pantothenic acid linked by cysteamine bridging groups. Pantethine treatment produced significant reduction of the better known risk factors (total cholesterol, LDL-cholesterol, triglycerides, and apo-B) and a significant increase of HDL-cholesterol (signally HDL2) and apolipoprotein A-I [13-14]. Vitamin B9 (Folic acid) 
supplementation reduces visceral obesity and improves plasma adiponectin levels [15]. Vitamin C (Ascorbic acid) reduced oxidative stress and markedly inhibited the development of experimental liver steatosis induced by choline-deficient diet [16]. Yin et al recently reported that the preventing effect of 1,25-dihydroxyvitamin $\mathrm{D}_{3}$ against high fat diet-induced hepatic steatosis in rats is related to the inhibition of lipogenesis and the promotion of fatty acids oxidation in rat liver [17]. In addition to the mentioned components, CIZ have many other micronutrients such as polyalcohols (mannitol and sorbitol), enzymes (amylase and lattase), inositol, and several sugars (maltose, maltotriose and maltitol). It is interesting to note that inositol, was previously shown to prevent fatty liver in rats [18]. All these beneficial effects of these micronutrients might play important roles in preventing the initiation and development of fatty livers. However, most of these micronutrients are removed in traditional food processing technology currently used in the world (extraction and refining), which will have an adverse effect on the hepato-protective effect.
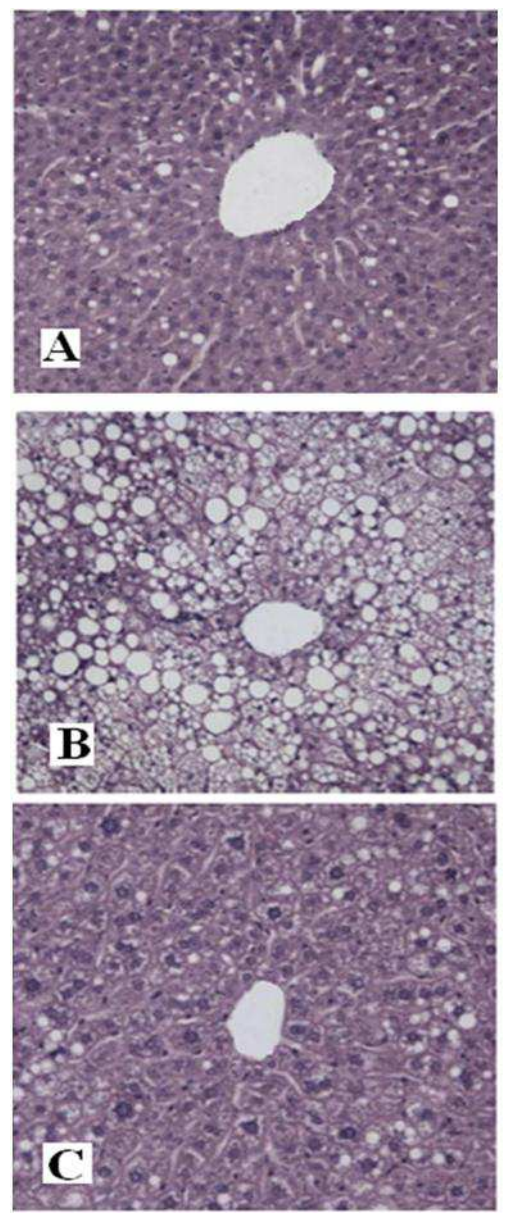

Figure 5. Histological sections of liver tissue from control, $x 150(A)$, HCD-fed, $x 100(B)$ and HCD-1:2CIZ, $x 200$ (C) C57BL6/N mice.

A significative decreased plasma ALT and AST activities, as well as plasma TCH levels, in comparison with the HCD-mice, who presented clear sign of metabolic steatosis, was observed. In the present study, solutions of CIZ supplemented to the HCD mice for 60 days resulted in a dose dependent reduction of $\mathrm{HCD}$-induced steatosis. Several studies have demonstrated that hepatocytes that internalize exogenous cholesterol repress its endogenous biosynthesis in response to HCD (19). Another important protective mechanism against hepatic cholesterol accumulation is cellular efflux of this steroid and bile acid biosynthesis $[20,1]$. This may explain the observed increased of plasma $\mathrm{TCH}$ in our HCD-fed mice. Furthermore, as expected, plasma activities of ALT and AST, were both substantially elevated by the HCD treatment. CIZ-treatment resulted in significant reductions in these plasma enzymatic activities in a dose dependent mechanism. The protective action of CIZ against hepatic steatosis involved not only ALT and AST activities, but also a decreased concentration of TAG. A recent study showed that the increased cholesterol ester in lipid droplets could limit the hydrolysis of triglycerides and decrease hepatic triglyceride secretion out of cells, leading to hepatic steatosis in the liver of HCD-fed mice [22]. The protective action of $\mathrm{CIZ}$ against $\mathrm{HCD}$-induced hepatic steatosis in mice appears to be likely mediated through the antioxidative property of some key micronutrients involved in regulation of lipogenesis. In conclusion obtained results showed a preventive and beneficial influence of CIZ supplementation on liver steatosis development.

\section{Conflict of Interest}

The authors have no potential conflicts of interest

\section{References}

[1] Cordain L, Eaton SB, Sebastian A, Mann N, Lindeberg S, Watkins BA, O'Keefe JH \& Brand-Miller J. Origins and evolution of the Western diet: health implications for the 21st century. Am J Clin Nutr. 2005; 81(2):341-354.

[2] Farrell GC \& Larter CZ. Nonalcoholic fatty liver disease: from steatosis to cirrhosis. Hepatology. 2006; 43(2 Suppl 1):S99-S112.

[3] Day CP \& James OF. Steatohepatitis: a tale of two "hits"? Gastroenterology. 1998; 114(4):842-845

[4] Kartal Ozer N, Negis Y \& Aytan N. Molecular mechanism of cholesterol or homocysteine effect in the development of atherosclerosis: role of vitamin E. Biofactors. 2003;19:6370

[5] Unger RH. Lipotoxic diseases. Annu Rev Med. 2002; 53:319-336

[6] Friis-Liby I, Aldenborg F, Jerlstad P, Rundström K \& Björnsson E. High prevalence of metabolic complications in patients with non-alcoholic fatty liver disease. Scand J. Gastroenterol 2004; 39: 864-869

[7] Mukhopadhyay-Sardar S, Rana MP \& Chatterjee M. Antioxidant associated chemoprevention by 
selenomethionine in murine tumor model. Mol Cell Biochem. 2000; 206(1-2):17-25

[8] Antonelli F \& Beninati S. Enhanced survival of B16-F10 melanoma tumour-bearing C57BL6/N mice treated with a mixture of antioxidants in: Recent Res Devel in Life Sci. 2011;5:51-60 Research Signpost, Trivandrum India.

[9] Torricelli P, Ferorelli P, De Martino A, Antonelli F \&Beninati S. Preventive Effects of A Mixture of Micronutrients with Antioxidative Properties on Experimentally Induced Prostate Hyperplasia. American J Life Sci. 2013; 1(1): 22-26.

[10] Yang SQ, Zhu H, Li Y, Gabrielson K, Trush MA \& Diehl AM: Mitochondrial Adaptations to Obesity-Related Oxidant Stress. Arch Biochem Biophys. 2000; 378(2):259-268.

[11] Curzio M, Esterbauer $\mathrm{H}$ \& Dianzani MU: Chemotacticactivity of hydroxyalkenals on rat neutrophils. Int J Tiss Reac 1985; 7:137-142.

[12] Noda S, Haratake J, Sasaki A, Ishii N, Umezaki H \& Horie A. Acute encephalopathy with hepatic steatosis induced by pantothenic acid antagonist, calcium hopantenate, in dogs. Liver. 1991;11(3):134-142.

[13] Bertolini S, Donati C, Elicio N, Daga A, Cuzzolaro S, Marcenaro A, Saturnino M \& Balestreri R. Lipoprotein changes induced by pantethine in hyperlipoproteinemic patients: adults and children. Int J Clin Pharmacol Ther Toxicol. 1986; 24(11):630-637.

[14] Osono Y, Hirose N, Nakajima K \& Hata Y. The effects of pantethine on fatty liver and fat distribution. J Atheroscler Thromb. 2000; 7(1): 55-58.

[15] Buettner R, Bettermann I, Hechtl C, Gäbele E, Hellerbrand C, Schölmerich J \& Bollheimer LC. Dietary Folic Acid
Activates AMPK and Improves Insulin Resistance and Hepatic Inflammation in Dietary Rodent Models of the Metabolic Syndrome. Horm Metab Res. 2010; 42(11): 769774 .

[16] Oliveira CP, da Costa Gayotto LC, Tatai C, Della Bina BI, Janiszewski M, Lima ES, Abdalla DS, Lopasso FP, Laurindo FR \& Laudanna AA. Oxidative stress in the pathogenesis of nonalcoholic fatty liver disease, in rats fed with a choline-deficient diet. J Cell Mol Med. 2002; 6(3):399-406.

[17] Yin Y, Yu Z, Xia M, Luo X, Lu X \& Ling W. Vitamin D attenuates high fat diet-induced hepatic steatosis in rats by modulating lipid metabolism. Eur J Clin Invest. 2012; 42(11):1189-1196

[18] Okazaki Y \& Katayama T. Effects of dietary carbohydrate and myoinositol on metabolic changes in rats fed 1, 1, 1trichloro-2,2-bis(p-chlorophenyl) ethane (DDT). J Nutr Biochem. 2003; 14: 81-9.

[19] Tabas I. Consequences of cellular cholesterol accumulation: basic concepts and physiological implications. J Clin Invest. 2002; 110(7):905-911.

[20] Tall AR, Costet P \& Wang N. Regulation and mechanisms of macrophage cholesterol efflux. J Clin Invest. 2002; 110 (7):899-904.

[21] Bjorkhem I. Do oxysterols control cholesterol homeostasis? J Clin Invest. vol. 2002; 110 (6):725-730.

[22] Alger HM, Brown JM, Sawyer JK, Kelley KL, Shah R, Wilson MD, Willingham MC \& Rudel LL. Inhibition of acyl-coenzyme A: cholesterol acyltransferase 2 (ACAT2) prevents dietary cholesterol-associated steatosis by enhancing hepatic triglyceride mobilization. J Biol Chem. 2010; 285(19):14267-14274. 